NIH STROKE SCALE $(P=0.86)$; however, the analysis was compromised by the statistical assumptions used. Compared with placebo, NXY-059 treatment resulted in more cases of complete neurological recovery $(P=0.01)$. NXY-059 did not alter survival, but patients who received this treatment plus alteplase were less likely to experience symptomatic hemorrhage than were those receiving placebo plus alteplase $(P=0.036)$.

The authors conclude that a confirmatory study is now required to verify the improvements in disability seen with NXY-059 treatment for acute ischemic stroke.

Rebecca Ireland

Original article Lees KR et al. (2006) NXY-059 for acute ischemic stroke. N Engl J Med 354: 588-600

\section{Mobile phone use does not increase risk of glioma}

The relationship between mobile phone use and incidence of intracranial malignancy has generated considerable research interest. The results of the largest ever case-control study on mobile phone use and risk of glioma have now been published in the BMJ.

The study, which is part of the INTERPHONE PROJECT, sampled 966 cases with low-grade or high-grade glioma and 1,716 control subjects from across the UK. Study participants were aged between 18 and 69 years. Cases had a first diagnosis of glioma between December 2000 and February 2004 and were selected from various treatment centers. Controls were randomly chosen from family physician patient lists to represent the general population. Cases and controls participated in a computerassisted personal interview, and a series of in-depth questions were used to determine the extent of mobile phone use.

The researchers found that mobile phone use did not significantly increase the risk of glioma, irrespective of cumulative number of hours of use, lifetime years of use, or cumulative number of calls made. Neither the location of phone use (urban versus rural) nor type of phone (analog versus digital) was related to risk of glioma. The study did reveal an increased risk of glioma ipsilateral to the side of the head that the phone was used and a decreased risk contralateral to side of phone use; however, these results were not substantiated in a subsequent analysis of handedness and the authors speculate that this effect could have been caused by a tendency of glioma patients to over-report phone use as being on the same side as their tumor.

\section{Alexandra King}

Original article Hepworth SJ et al. (2006) Mobile phone use and risk of glioma in adults: case-control study. BMJ [doi: 10.1136/bmj.38720.687975.55]

\section{Possible role for leptin in treating depression}

Most studies investigating the adipocyte hormone, leptin, have focused on its appetiteregulating activity in the hypothalamus. Leptin receptors are also found in brain structures involved in emotional and mood regulation, however, such as the hippocampus. Support for a possible role of leptin in modulating mood is provided by a new animal study, which found that plasma leptin levels of rats chronically subjected to social defeat or unpredictable stress were lower than those of controls. Leptin injections reversed the 'anhedonic' behaviors of these rats.

The antidepressant-like effects of leptin were evaluated by monitoring sucrose preference and by the forced-swimming test. In both tests, leptin's effects were comparable to those of conventional antidepressants. Interestingly, however, different behaviors were induced by leptin and the antidepressant desipramine, respectively, in the forced-swimming test: desipramine was associated with climbing activity whereas leptin was associated with longer duration of swimming. This difference was not attributable to leptin's energy-regulating activity; comparison of the effects of infusing leptin directly into different brain areas revealed that hippocampal infusion produced similar antidepressant-like effects to systemic treatment, whereas hypothalamic infusion produced no such effects.

Rising plasma leptin levels have been observed in patients who were responding to conventional antidepressant treatment, although depressed patients do not always have lower leptin levels than healthy individuals. It might be that leptin levels are low only in a subset of depressed patients.

Caroline Barranco

Original article Lu X-Y et al. (2006) Leptin: a potential novel antidepressant. Proc Natl Acad Sci USA 103: 1593-1598

\section{GLOSSARY}

NIH STROKE SCALE

A structured, clinician-rated scale incorporating physical examination, pre-set questions, and language assessment to quantify neurological deficits following stroke

\section{INTERPHONE PROJECT}

An international

collaborative study

investigating the relationship between mobile phone use and risk of intracranial malignancy 